\title{
EL PROCESO ELECTORAL MEDIADO POR TWITTER EN ECUADOR: EL CASO DEL REFERENDUM Y LA CONSULTA POPULAR DE FEBRERO DE 2018
}

\section{ELECTORAL PROCESS MEASURED BY TWITTER IN ECUADOR: CASE OF THE REFERENDUM AND THE PEOPLE'S CONSULTATION OF FEBRUARY 2018}

Gary Lenin Pulla Zambrano, Mgs. Máster Universitario en Dirección Estratégica e Innovación en Comunicación (España). Docente de la Facultad de Marketing y Comunicación de la Universidad Tecnológica ECOTEC, Ecuador. gpulla@ecotec.edu.ec

Ana María Almansa Martínez, PhD. Doctora en Comunicación (España). Profesora de la Universidad de Málaga, España. anaalmansa@uma.es

\section{ARTÍCULO DE INVESTIGACIÓN}

Recibido: 10 de septiembre de 2018.

Aceptado: 8 de noviembre de 2018.

\section{RESUMEN}

El uso de las redes sociales ha ganado relevancia como parte de la estrategia de comunicación en la promoción electoral de candidatos y propuestas en torno a elecciones de diferente índole. En este estudio se analiza la interacción de los votantes en la red social Twitter, y se plantea como principal objetivo de investigación el análisis de las características de los mensajes emitidos por los partidos y organizaciones sociales calificados para hacer promoción dentro del marco de la campaña electoral de la Consulta Popular de 2018 en Ecuador. Bajo esta premisa se utilizó la técnica de análisis de contenido para analizar al mismo tiempo los 5 tweets que recibieron más re tweets y los 5 que recibieron más favoritos por cada partido u organización. Los resultados resaltaron la importancia del uso de fotos y videos como foco de atención, así como hashtags como un atributo que se repitió sin importar el partido u organización. Se concluyó que unos de los principales enfoques en cuanto a estrategias de comunicación política para redes sociales debe centrarse en los recursos 
visuales y acciones realizadas in situ, además de mantener siempre una referencia clara hacia la propuesta que se desea impulsar.

Palabras clave: Twitter; redes sociales; comunicación política; campañas electorales; consulta popular

\section{ABSTRACT}

The use of social networks has gained relevance as part of the communication strategy in the electoral promotion of candidates and proposals around different kinds of elections. This study analyzes the interaction of voters in the social network Twitter, and considers as the main research objective the analysis of the characteristics of messages issued by parties and social organizations qualified to promote within the framework of the electoral campaign of the Popular Consultation of 2018 in Ecuador. Under this premise, the content analysis technique was used to analyze at the same time the 5 tweets that received the most tweets and the 5 that received the most favorites for each game or organization. The results highlighted the importance of using photos and videos as a focus, as well as hashtags as an attribute that was repeated regardless of the party or organization. It was concluded that one of the main approaches in terms of political communication strategies for social networks should focus on visual resources and actions carried out in situ, in addition to always maintaining a clear reference to the proposal that is to be promoted.

Keywords: Twitter; social media; political communication; electoral campaigns; referendum

\section{INTRODUCCIÓN}

La interacción, marcada por la llegada de las redes sociales, es un tema de investigación de la última década, donde se evidencia la participación de diferentes actores, siendo el ámbito social, político y económico, los de mayor notoriedad a nivel mundial. Esta nueva forma de comunicarse, caracterizada por la inmediatez y la actualización constante de la información ha permitido la evolución del usuario pasivo, receptor de información, a un usuario comprometido con la comunidad en la que participa y en la que se desenvuelve a través de los medios digitales como Facebook y Twitter, convirtiéndose en un usuario prosumidor de 
información. Así, se van formando pequeñas redes de comunicación para luego formar parte de la red global.

En el ámbito de la comunicación política, se ha incursionado en el uso de las redes sociales como medio de propagación de los mensajes que los políticos entregan a la sociedad. Una referencia de esta forma de comunicación para la generación de actividades en comunidades, es la campaña electoral de Barack Obama en el año 2008, que resaltó como pionera en el uso de canales digitales para la difusión de información referente a sus propuestas de gobierno y que fue de gran aporte para los resultados electorales a su favor. Así como el caso de Obama en Estados Unidos, el uso de los medios digitales se ha normalizado como un estándar a nivel global en términos de uso y gestión. De esta manera, se perciben partidos, candidatos y movimientos que cuentan con perfiles en las redes sociales de mayor tráfico para abarcar un público cuyo medio de información cotidiano es la red. Esta modalidad de hacer política a través de los medios digitales se puede evidenciar en el contexto local, sobre todo en el período electoral del 2017, donde todos los candidatos a la presidencia de Ecuador contaban, de manera legal, con perfiles en las redes sociales Facebook y Twitter, haciendo uso correcto de las políticas de seguridad en internet y de todo aquello que conllevan las normas de uso de las mismas. Sin embargo, las estrategias utilizadas para la publicación de la información fueron diferente entre un partido y otro. En este aspecto, se reflejaron deficiencias en cuanto al aprovechamiento del potencial de estos medios para captar la atención de los internautas, lo que influyó en la determinación y la percepción del público de estas redes para la toma de decisiones sobre la elección de un candidato.

En Ecuador, tras las elecciones presidenciales del 2 de abril de 2017, el presidente electo Lenin Moreno instó a la ciudadanía a participar de una consulta popular y referéndum para realizar cambios en la constitución ecuatoriana, donde se esperaba que el público votante respalde reformas definidas, por el periódico "El Comercio" (2018) por temas que se resumen a continuación:

- $\quad$ Referendum pregunta 1: Lucha contra la corrupción

- $\quad$ Referendum pregunta 2: Reelección indefinida

- Referendum pregunta 3: Consejo de participación ciudadana

- $\quad$ Referendum pregunta 4: Protección a la niñez

- $\quad$ Referendum pregunta 5: Minería 
- $\quad$ Consulta popular pregunta 1: Plusvalía

- $\quad$ Consulta popular pregunta 2: Yasuní

Esta propuesta fue aprobada por el Consejo Nacional Electoral del Ecuador y el 4 de febrero de 2018 se llevó a cabo el proceso electoral, en el cual se contó con una fuerte campaña política sostenida por el gobierno a favor de un voto positivo, la misma que tuvo gran relevancia en las redes sociales Facebook y Twitter. En esta campaña se inscribieron alrededor de 40 organizaciones políticas, las mismas que tuvieron presencia en la red, destacando la participación de los partidos políticos que apoyaron la propuesta del gobierno. De los partidos políticos que manifestaron su opinión sobre la consulta mediante las redes sociales Facebook y Twitter, el partido Foro Nacional de Mujeres no apoyó la propuesta gubernamental.

De acuerdo a los resultados, se demuestra que las redes sociales influyen en las decisiones de los internautas. En la campaña política realizada para que la consulta popular tuviera una aceptación masiva a favor de la propuesta de gobierno, representada por el SI, se observó que la cobertura de información en las redes sociales fue masiva y logró cubrir todos los estratos sociales en el país. La información difundida a través de estos medios se realizó mediante videos, caricaturas, texto, entre otros y consiguió que la ciudadanía comprenda las razones por el cual era necesario realizar dichas reformas.

Con este antecedente, en este artículo se analiza el uso de las redes sociales, especialmente de twitter, que utilizaron estas organizaciones políticas con el fin de apoyar una campaña electoral.

\section{REVISIÓN TEÓRICA}

\subsection{Social Media}

Se consideran las aproximaciones hacia el significado de social media planteado por Mandiberg (2012), quien asocia conceptos generales planteados por la convergencia de diversos autores como Jenkins, Rosen y Benkler, para definirlos como un proceso influenciado por audiencias colabortivas que producen información abierta al público y contenido siempre orientado al contexto de la web.

Es bajo la premisa de asistencia y cooperación que se desarrollan las comunidades digitales, pues bajo el concepto de humanización que ahora maneja, se divisan ciertas características 
que han venido a adoptar. Estas características, planteadas por Marquina (2012) se destacan a continuación:

- $\quad$ Deseo de interactuar para satisfacer necesidades

- Colaboración de un proposito que conlleva la razón de ser de la comunidad

- Disposición de un sistema informático que media interacciones y facilita cohesión

Además de las comunidades, el autor también nombra la aparición de las redes sociales, que las define como redes virtuales que permiten conectar a personas con sus conocidos, familiares, amigos o desconocidos con el fin de compartir contenidos, interactuar y crear comunidades acerca de intereses similares.

\subsection{Redes sociales como herramienta de interacción}

Se sustenta que las redes sociales han revolucionado el concepto clásico de relación social, desde que permiten un conglomerado de actividades y ventajas como las define Caldevilla (2010):

- Modifican las fórmulas de redacción y periodismo clásico, permitiendo información de manera inmediata.

- $\quad$ Se puede conocer gente que comparte nuestros intereses y colaborar con ellos

- Han propiciado el surgimiento de grupos de cooperación y manifestaciones a nivel mundial.

- $\quad$ Posibilitan desarrollar aficiones y formar parte de diversas comunidades.

- $\quad$ Permiten una retroalimentación informativa casi instantánea.

Con esto, se logra constituir interactividad con los grupos de interés y consumidores de una marca, que ahora, cada vez más acostumbrados al uso de los medios digitales, la avizoran como la siguiente fuente de realizar transacciones humanas que incluyan la escucha de sus necesidades o demandas, que pueden servir de información para fines sociales, empresariales o políticos. Actualmente existen diferentes redes sociales en las que pueden realizarse estas actividades, y cabe acotar que dichas redes tienen diferente acogida acorde al contexto de uso en cada país. Localmente, el estudio realizado por IAB Ecuador (2017) 
acerca del consumo de las redes sociales indica, en la figura 1, que las redes más utilizadas a nivel país son: Facebook, Instagram, Twitter y Whatsapp.

Figura 1. Uso de redes sociales en Ecuador 2017.

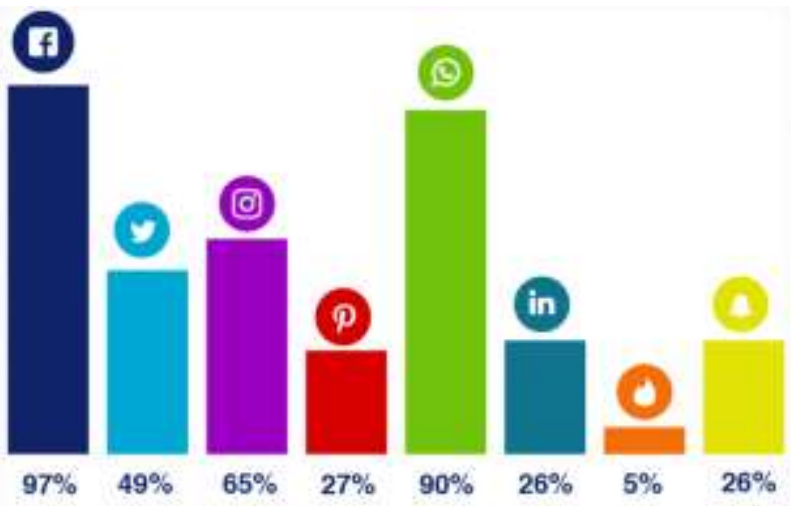

Fuente: IAB Ecuador, 2017.

\subsection{Ciberpolitica y Twitter en Ecuador}

Caballero (2016) define a la ciberpolítica como las "nuevas formas de interacción entre el gobierno y los ciudadanos, entre éstos y los partidos políticos, entre las empresas y los órganos legislativos, entre ciudadanos de un régimen político y entre ciudadanos del mundo", dejando claro el progreso del uso de los medios online en los actores que se mencionan en la definición planteada.

Twitter, por su parte, fué adoptado como un nuevo espacio de socialización de ideas e interacción con mensajes cortos que se actualizaban muy rapido y a los cuales el usuario podía suscribirse (Small, 2010). En el contexto local, fue esta red social la que mejor se logró posicionar luego de la crisis mencionada en el apartado anterior, pues "Twitter es una herramienta paralela a la versión periodística de los medios de comunicación en relación a su gestión y participació pública, consolidando nuevos espacios de difusión y debate" (Marín, Punin, Hinojosa, \& Ruiz, 2016)

En el caso del uso de nuevas formas de interacción digital nivel local, se puede señalar la crisis política ocurrida el 30 de septiembre de 2010, cuando la policía se levanto contra el gobierno, marcó un hito en el uso de redes sociales, popularizandolas y posicionandolas 
como herramientas de información masiva supliendo la falta de información inmediata de los medios de comunicación tradicionales (Coronel \& Mier, 2011). Durante ese día, Twitter y las cuentas de periodistas locales se convirtieron en una fuente de información que se planteó como un recurso constante de todos los eventos que iban ocurriendo al momento.

Asímismo, el uso de las redes durante campaña electoral del 17 de febrero de 2013, para elegir presidente del Ecuador, se evidenció con claras iniciativas por parte de los candidatoss y sus equipos de comunicación para contar con presencia digital, como lo asevera Escandón (2013).

En la campaña más reciente, ocurrida para las elecciones presidenciales de 2017, se evidenció que cada uno de los candidatos contaba con presencia, no solo en redes sociales, sino también en medios como blogs y webs referentes a su propuesta, concluyendo que "candidatos como Guillermo Lasso, Lenin Moreno, Paco Moncayo y Cynthia Viteri, todos pertenecientes a partidos políticos con poder y experiencia, además de ser figuras políticas con trayectoria en elecciones pasadas, tuvieron un mejor desempeño que otros candidatos" (Pulla, 2017).

Aún así, Albornoz y Rosales (2012) indican que "Si bien fueron los ciudadanos quienes más opinaron e informaron en Twitter ... de los tweets publicados por ellos, sólo $44 \%$ eran informativos, contra $51 \%$ de opinión y $5 \%$ de opinión-información”. Esta evaluación permitió conocer que los ciudadanos intervinieron como productores de información, y que no todos los tweets contaban con la veracidad de un medio tradicional, pues en gran medida también se incluían opiniones que no necesariamente se conformaban como periodismo ciudadano. La presente investigación busca refrescar la información obtenida en investigaciones anteriores y conocer como se desempeñan actualmente los partidos políticos y movimientos en la red social Twitter.

\section{MATERIALES Y MÉTODOS}

Se proponde el uso del modelo LOOP, elaborado por Pulla (2017) para identificar actores y actividades principales de la comunicación en redes sociales a través de métricas que permiten obtener resultados cuantitativos y cualitativos sobre el desempeño de la campaña y el perfil de los partidos y movimientos en estudio, descrito en el gráfico 2 a continuación: 
Figura 2. Modelo de análisis estratégico de marca LOOP.

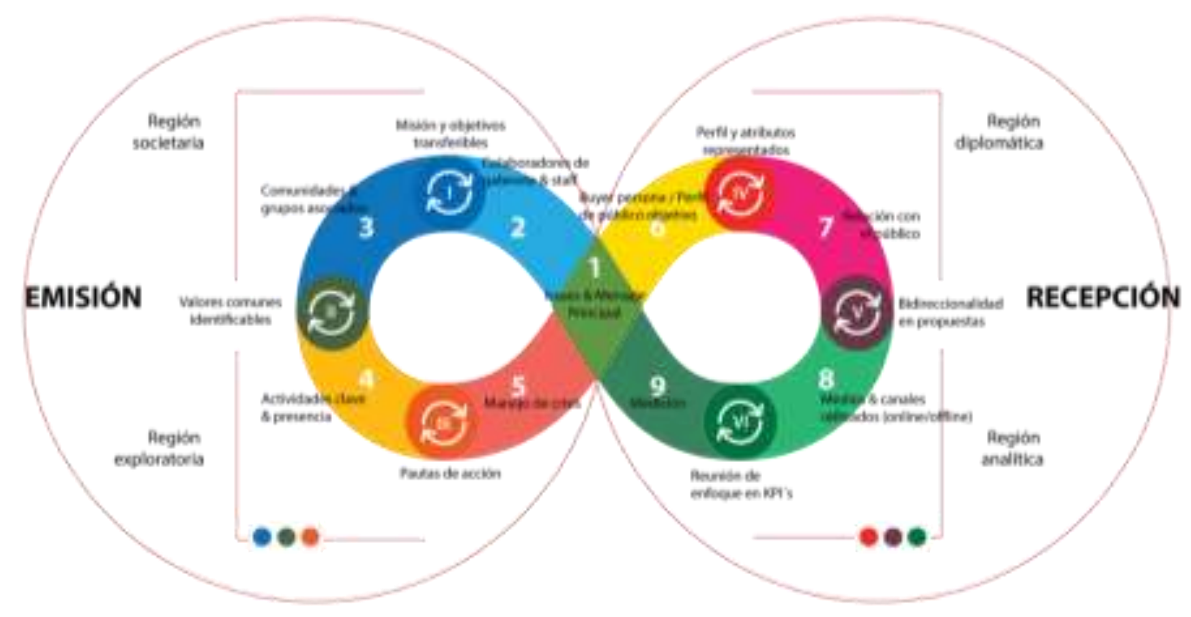

Fuente: Pulla, 2017.

El modelo comprende 2 macro-componentes divididos en la emisión y recepción de información. El primer macro-componente que se define es establecido en el rol de Emisor porque todos los elementos que lo componen derivan de acciones propias del emisor. Este se describe en la tabla 1 a continuación:

Tabla 1. Elementos del macro-componente de Emisor del modelo LOOP.

\begin{tabular}{ll}
\hline Elemento & Descripción \\
\hline Issues y mensaje principal & Similar a cualquier campaña, el primer paso que se \\
& debe dar es la definición del mensaje o issues que \\
& va a recoger el usuario, en este caso, el candidato \\
& político y que va a servir de portaestandarte para \\
& toda la estrategia llevada a cabo en la campaña \\
\hline Colaboradores de gabinete y staff & Este bloque comprende todo el personal por el que \\
& se compone el gabinete de comunicación online o \\
& el staff de comunicaciones online. El diseño, cuadro \\
& de mando y conformación del mismo dependerán \\
& en su totalidad de los criterios del usuario \\
& representado, así como de los resultados que \\
& presente.
\end{tabular}




\begin{tabular}{ll}
\hline Comunidades y grupos asociados & Abarca y enumera a todo colectivo o agrupación \\
& que brinde apoyo comunicacional a la marca. En \\
& este caso al candidato político, y se puede referir a \\
& otros movimientos políticos, grupos de interés \\
& religiosos, ONGs, etc... \\
\hline Actividades clave y presencia & El bloque busca identificar el número de canales \\
& online, ya sean redes sociales, blogs, podcasts u \\
& otros que el candidato tiene y también comprende \\
& el verificar si mantiene dichos canales con \\
& constante actividad según la naturaleza del mismo. \\
El presente apartado tiene como finalidad la \\
generación de un plan de acción dirigido hacia \\
cualquier situación que ponga en duda la reputación \\
del candidato
\end{tabular}

Fuente: Elaboración propia.

Por otro lado, los bloques de la recepción que se compone del target, mensaje entregado y medición de actividades. Estos bloques se describen en la tabla 2 presentado a continuación:

Tabla 2. Elementos del macro-componente de Recepción del modelo LOOP.

\begin{tabular}{ll}
\hline Elemento & Descripción \\
\hline Buyer persona/ Perfil del público objetivo & La sección enumerada busca recopilar el \\
& conjunto de atributos que mejor representen \\
& al público objetivo con el fin de conocer no \\
& solo sus rasgos sino también las \\
& actividades que realiza cada cierto tiempo y \\
& así poder generar un mayor y mejor \\
& impacto. \\
& El bloque de relación con el público no dista \\
Relación con el público & de su similar descripción en el Canvas \\
& Business Model, porque al igual que este, lo \\
& que se busca es analizar en qué tonos \\
& mantiene dialogo con los electores
\end{tabular}


ecuatorianos y qué tipo de recursos (videos, posts, fotos, etc...) utiliza para generar interacción con él de manera positiva.

Medios y Canales usados

Medición
El presente bloque determina

específicamente cuáles son los medios de mayor relevancia por parte del emisor para

la campaña llevada cabo

El principal objetivo de este bloque será la de recopilar todos los datos obtenidos en todas las plataformas sociales para la evaluación general de desempeño que se genera alrededor de los canales utilizados.

Fuente: Elaboración propia.

De la presente metodología se buscará obtener solamente los datos vinculantes a la recepción, excluyendo también los campos de eslabones. En consecuencia, el modelo utilizado para la presente investigación se expone en la imagen 3, pues existen los medios para obtener resultados reales de estos componentes.

Figura 3. Modelo de análisis estratégico adaptado a la investigación planteada.

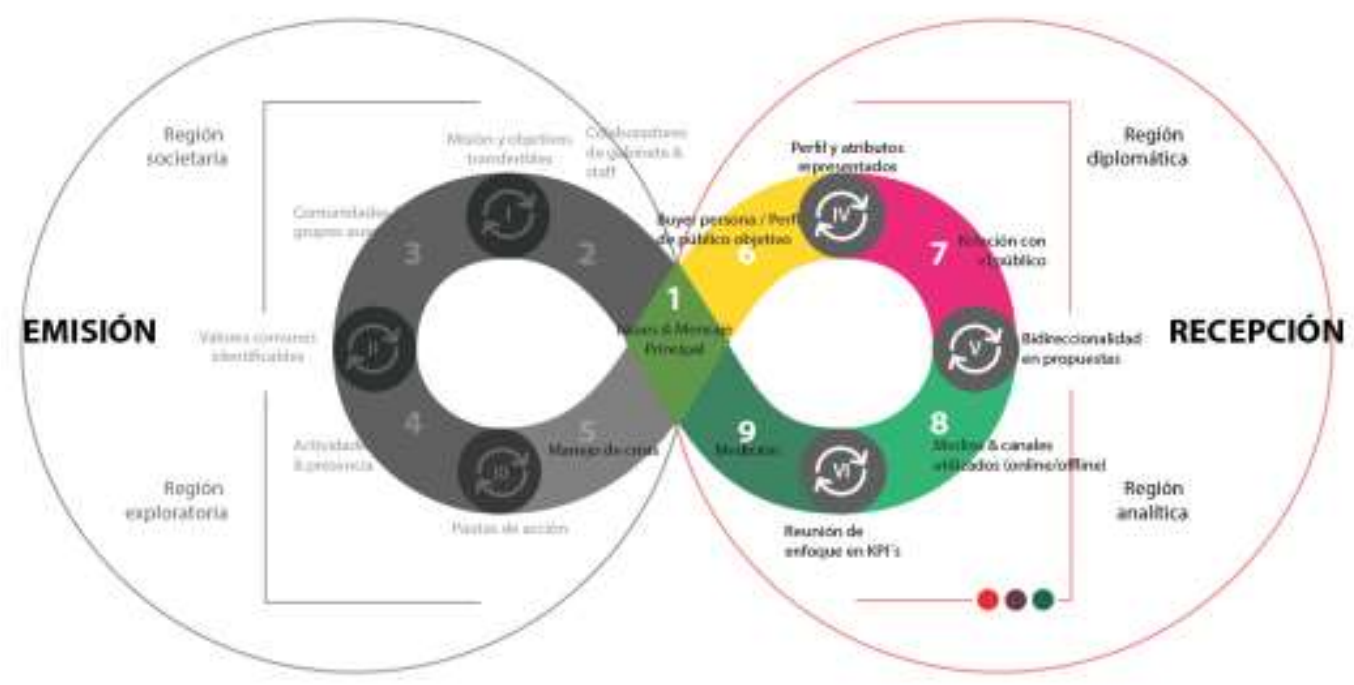

Fuente: Elaboración propia.

\subsection{Diseño de la investigación}


La presente investigación tiene un diseño cuasi-experimental, de alcance descriptivo y de corte transversal. Los elementos de la recepción se trabajarán con una metodología mixta, es decir, cualitativa y cuantitativa. Igualmente, se utilizará la herramienta de analítica de redes sociales Twittonomy para complementar los resultados del estudio, los mismos que se evaluarán con estadística descriptiva. A continuación, se presenta en la tabla 3 las técnicas de investigación e instrumentos a ser utilizados:

Tabla 3. Técnicas e instrumentos de investigación.

\begin{tabular}{|c|c|}
\hline Bloque & $\begin{array}{l}\text { Técnicas de Instrumentos } \\
\text { investigación }\end{array}$ \\
\hline Issues y mensaje principal & $\begin{array}{l}\text { Este bloque solamente puede tener } 2 \\
\text { mensajes, que serán las posturas del partido o } \\
\text { movimiento frente a la campaña por el sí o por } \\
\text { el no }\end{array}$ \\
\hline Buyer persona/ Perfil del público objetivo & $\begin{array}{l}\text { Reportes de Twittonomy } \\
\text { herramientas sociales }\end{array}$ \\
\hline Relación con el público & Análisis de contenido \\
\hline Medios y Canales usados & $\begin{array}{l}\text { Solamente se ha considerado Twitter como red } \\
\text { social a ser analizada }\end{array}$ \\
\hline Medición & $\begin{array}{lrr}\text { Reportes } & \text { de } & \text { Twittonomy } \\
\text { herramientas } & \text { de } & \\
\text { análisis de } & \text { redes } \\
\text { sociales } & \end{array}$ \\
\hline
\end{tabular}

Fuente: Elaboración propia.

A partir de los requerimientos propios de la investigación, se hizo uso de reportes provenientes de Twittonomy, una herramienta de analítica web enfocada a esta plataforma y que es alimentada tanto por Bit.ly como por por Twitter, pero que no cuenta con el desarrollo ni algún tipo de afiliación a estas fuentes (Diginomy Pty Ltd, 2017).

El análisis de contenido, por otro lado, se llevó a cabo bajo un esquema de plantilla basado en el modelo de Almansa (2015), y Small (2010), quienes trabajaron en investigaciones del mismo perfil y cuyas fichas contienen apartados de importancia para esta investigación. De 
esta manera, se recopiló información de los 5 tweets con más interacción en forma de retweets y favoritos, y se les aplicó una nueva plantilla mostrada en la tabla 4 a continuación:

Tabla 3. Plantilla utilizada para el análisis de contenido.

Nombre del partido

General

No. Tweets

Tweets por día

$\%$ Conversaciones

Hashtags usados

Nivel de interacción según recursos

\section{Enlaces}

Videos

Fotos

Texto

\section{Uso de recursos de la herramienta}

\section{Menciones}

\section{Hashtags}

\section{Mencion \& hashtag}

No se uso

Fuente: Elaboración propia

\section{ANÁLISIS DE RESULTADOS}

El número de oganizaciones políticas y movimientos inscritos en su convocatoria para hacer campaña electoral apoyando el Si o el No tuvo 30 inscritos en una primera instancia. Sin embargo, este número subió luego de reabrir el proceso de registro electoral quedando registradas 40 organizaciones. A partir de la publicación oficial de los inscritos, se realizó la busqueda de sus cuentas en twitter y solamente se encontraron 21 cuentas de las 36 organizaciones o movimientos que apoyaban el Si. Igualmente se realizó la busqueda de las cuentas sociales de las 4 organizaciones que apoyaron el No, de las cuales solo se evidenció 1 cuenta activa. 
A partir de estos resultados obtenidos, se utilizó la herramienta Twittonomy para analizar la información emitida por estas cuentas durante la campaña electoral y se obtuvo como resultado que apenas 8 movimientos, todos vinculados a la propuesta del Si habían realizado una difusión contínua de mensajes en redes sociales, como se muestra en el gráfico 1.

Gráfico 1. Cantidad de Tweets durante la campaña electoral.

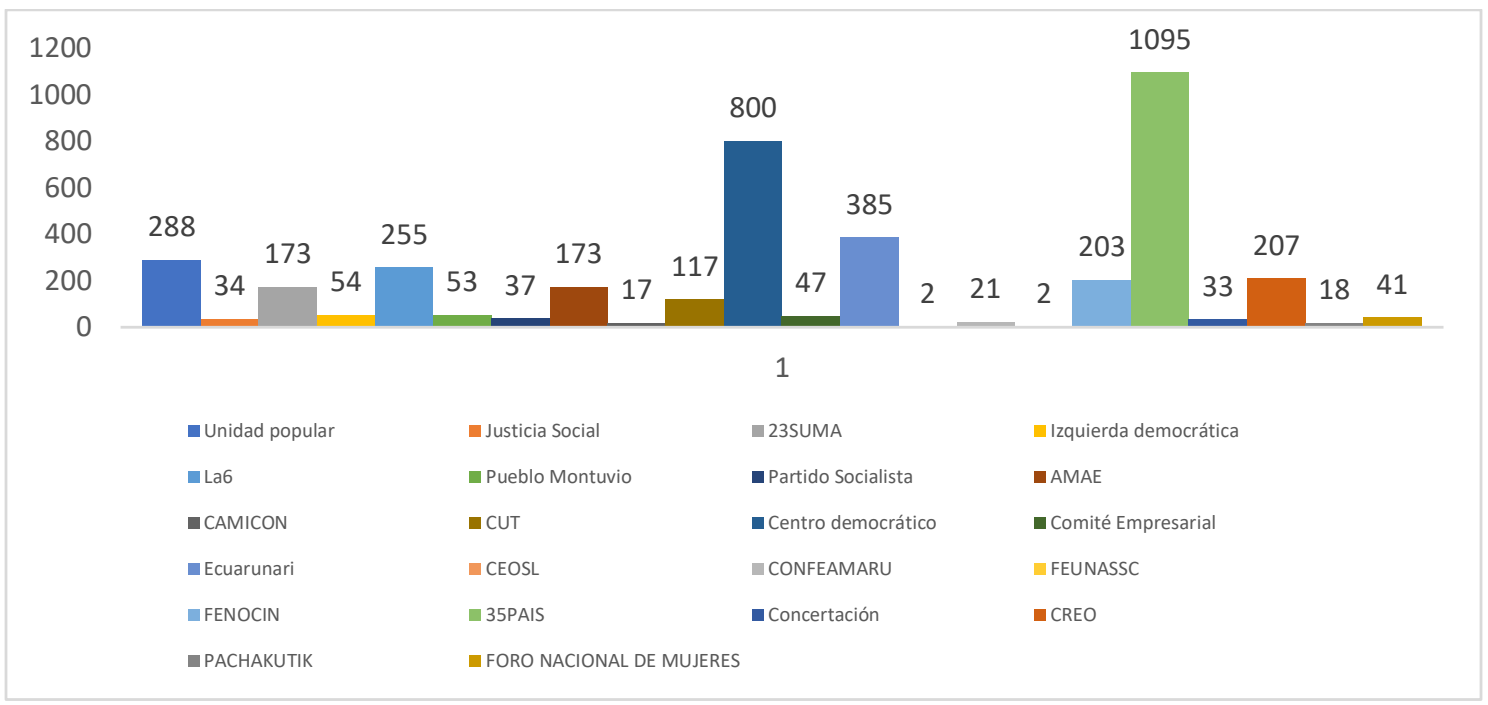

Fuente: Elaboración propia

Por conseguiente se consideraron para el análisis solamente las cuentas de los partidos y movimientos que contaran con más de 150 Tweets para no incluir datos que no serían de mayor utilidad en la investigación. Bajo esta condición, en la tabla 1 se enumeran los partidos elegidos para el análisis con el número de tweets correspondientes desde el 4 de enero hasta el 1 de febrero de 2018, espacio en el que se desarrollo la campaña electoral.

Tabla 4. Partidos y movimientos, con mayor cantidad de participación, que apoyaron el Si en la campaña electoral.

\begin{tabular}{ll}
\hline Partido o movimiento & Número de Tweets enviados en campaña \\
\hline Unidad popular & 288 \\
La 6 & 255 \\
23SUMA & 173 \\
AMAE & 173
\end{tabular}




$\begin{array}{ll}\text { Centro Democrático } & 800 \\ \text { Ecuarunari } & 385 \\ \text { FENOCIN } & 203 \\ \text { 35PAIS } & 1095 \\ \text { CREO } & 207\end{array}$

Fuente: Elaboración propia

\subsection{Perfil del público}

Se realizó un analisis del perfil de público considerando 2 variables obtenidas en Twittonomy. En primer lugar, se consideró los días de la semana en que se realizó mayor cantidad de interacciones, siendo estos días los lunes, miercoles y jueves, como se describe en el gráfico 2.

Gráfico 1. Tweets de los partidos y movimientos políticos por semana.

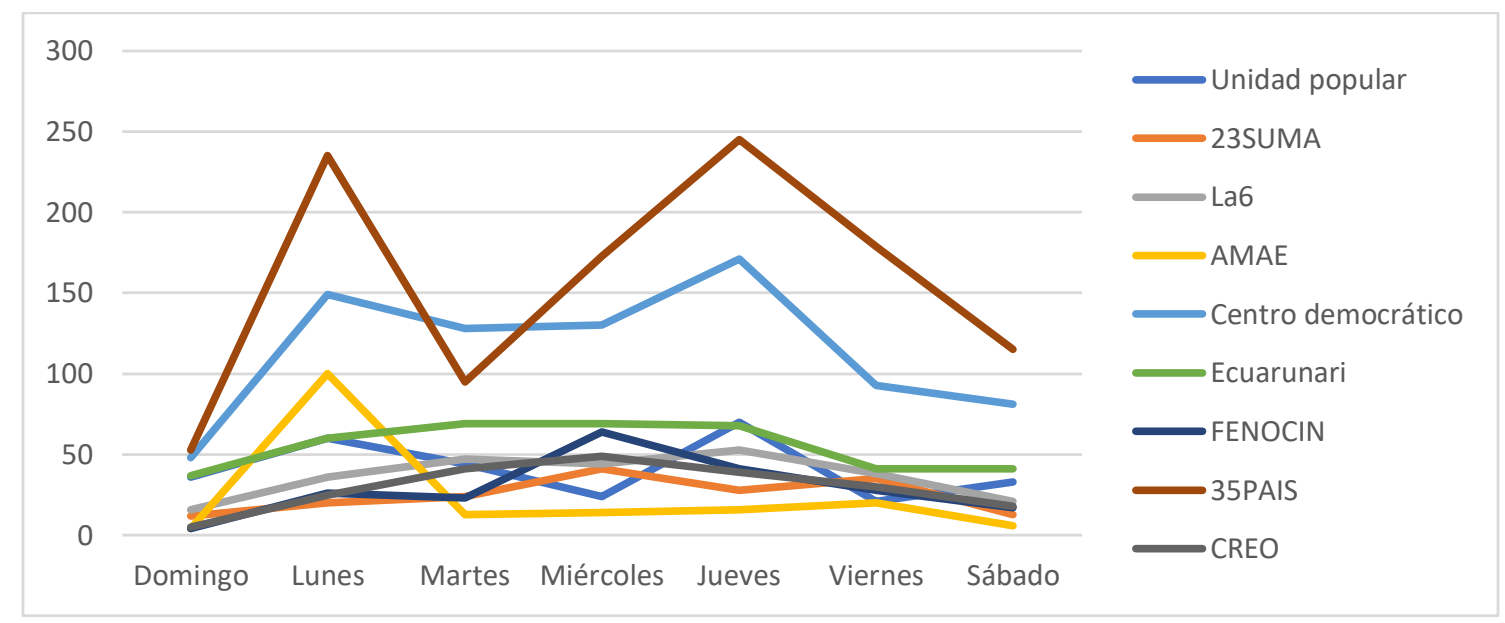

Fuente: Elaboración propia.

Adicionalmente, la herramienta utilizada también permitió conocer la interacción de las personas durante las diferentes horas del día, para lo que se procedió a aglomerar todas las cuentas con el fin de totalizar el número de tweets que generaron las diferentes cuentas a lo largo del día durante toda la campaña. Así, podemos destacar en el gráfico 3 que los mayores picos de actividad a la hora de twittear fueron entre las franjas horarias de $12 \mathrm{~h} 00$ a $02 \mathrm{~h} 00 \mathrm{y}$ de $16 \mathrm{~h} 00$ a $18 \mathrm{~h} 00$.

Gráfico 2. Horarios de las publicaciones. 


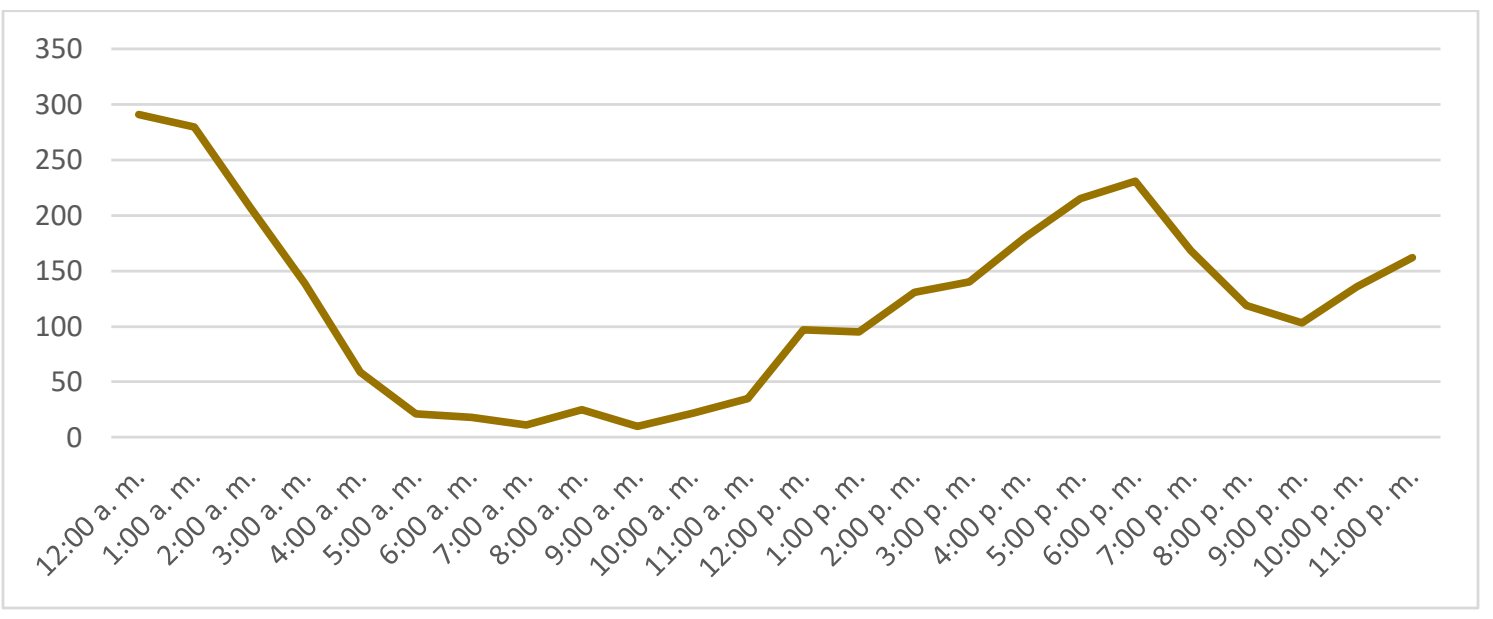

Fuente: Elaboración propia

Con estos resultados se puede corroborar que el perfil del público aún se mantiene en una tendencia de publicación de horarios vespertinos y nocturnos, haciendo referencia a un perfil de público que posiblemente cumple con jornadas laborales y no tiene tiempo durante el día, a más de un momento en el almuerzo para, poder revisar sus redes sociales, pero que sí se vuelve activo de noche e incluso en la madrugada.

\subsection{Relación con el público}

En la tabla 7 se muestra el tipo de contenido que tuvo mayor repercusión tanto en los tweets que más obtuvieron retweet como en los que tenían más Favoritos. Estos resultados En el caso de los Retweets, los recursos que más se utilizaron fueron las fotos y en el caso de los Favoritos, el recurso que más se utilizó fueron los videos. Además, en la cuenta de @23SUMA se evidenció solamente el uso de textos que tuvieron un gran número de retweets y favoritos, por lo que se evidencia que no solamente el uso de los recursos gráficos puede impactar a los internautas o que existe un gran apoyo gestionado de los usuarios afines al partido que apoyaron conscientemente ese tweet. 
Tabla 5. Tipo de contenidos mostrados en las cuentas de los partidos y movimientos en campaña.

\begin{tabular}{lll}
\hline Partido o movimiento & RT & Favoritos \\
\hline Unidad popular & Foto & Video \\
\hline LA 6 & Foto & Foto \\
\hline 23SUMA & Texto & Texto \\
\hline AMAE & Foto & Foto \\
\hline Centro Democrático & Enlace & Foto \\
\hline Ecuarunari & Video & Video \\
\hline FENOCIN & Foto & Foto \\
\hline 35PAIS & Video & Video \\
\hline CREO & Video & Video
\end{tabular}

Fuente: Elaboración propia

Con este resutado también se evidencia que la mayoría del público que se vinculó a las cuentas de los partidos y movimientos se vieron influenciados en gran medida por el recurso visual y lúdico que podría haber sido representado mediante imágenes o grabaciones con el tema principalmente vinculado al Si/ No de la campaña.

\subsection{Medición}

Con el fin de complementar la parte de la emisión, se utilizó nuevamente la herramienta twittonomy con información referente a los tweets durante la campaña de los partidos y movimientos descritos anteriormente y se obtuvieron los resutlados de la tabla 7.

Tabla 6. Medición general del desempeño en campaña.

\begin{tabular}{|c|c|c|c|c|c|c|c|c|}
\hline $\begin{array}{l}\text { Unida } \\
\text { d }\end{array}$ & $\begin{array}{l}\text { 23SU } \\
\text { MA }\end{array}$ & La6 & AMAE & $\begin{array}{l}\text { Centr } \\
\text { o }\end{array}$ & $\begin{array}{l}\text { Ecuar } \\
\text { unari }\end{array}$ & $\begin{array}{l}\text { FENO } \\
\text { CIN }\end{array}$ & $\begin{array}{l}\text { 35PAl } \\
\mathrm{S}\end{array}$ & CREO \\
\hline popul & & & & demo & & & & \\
\hline ar & & & & $\begin{array}{l}\text { crátic } \\
0\end{array}$ & & & & \\
\hline
\end{tabular}




\begin{tabular}{llllllllll}
$\begin{array}{l}\text { Total } \\
\text { tweets }\end{array}$ & 288 & 173 & 255 & 173 & 800 & 385 & 203 & 1095 & 207 \\
\hline $\begin{array}{l}\text { Tweets } \\
\text { per day }\end{array}$ & 9,93 & 5,97 & 8,79 & 5,97 & 27,59 & 13,28 & 7 & 37,76 & 7,14 \\
\hline $\begin{array}{l}\text { Retwee } \\
\text { ts }\end{array}$ & 217 & 170 & 254 & 96 & 150 & 105 & 53 & 804 & 162 \\
\hline $\begin{array}{l}\text { User } \\
\text { mentio }\end{array}$ & 29 & 0 & 3 & 494 & 696 & 28 & 1288 & 142 & 4 \\
ns & & & & & & & & & \\
\hline $\begin{array}{l}\text { Replie } \\
\text { s }\end{array}$ & 0 & 0 & 44 & 63 & 2 & 25 & 0 & 0 \\
\hline Hashta & 107 & 0 & 1 & 0 & 1163 & 122 & 0 & 546 & 23 \\
gs & & & & & & & & & \\
\hline
\end{tabular}

Fuente: Elaboración propia

A partir de estos resultados, durante el tiempo de campaña la atividad de ciertos partidos y movimientos tuvo mucho mayor impacto que el de otros. A partir de esto, se pudo confirmar que grupos con experiencia definida en manejo de campañas digitales como Centro Democrático, CREO, La6 o 35PAIS tuvieron un desempeño mucho más constante y formidable que otros grupos que realizaron autogestión. En consecuencia, se puede denotar una mayor cantidad de tweets por día, así como retweets, siendo estos 4 exponentes los que abarcaron más cantidad de contenido retweeteado, como se muestra en el gráfico 4.

Gráfico 3. Porcentaje de tweets que fueron retweet.

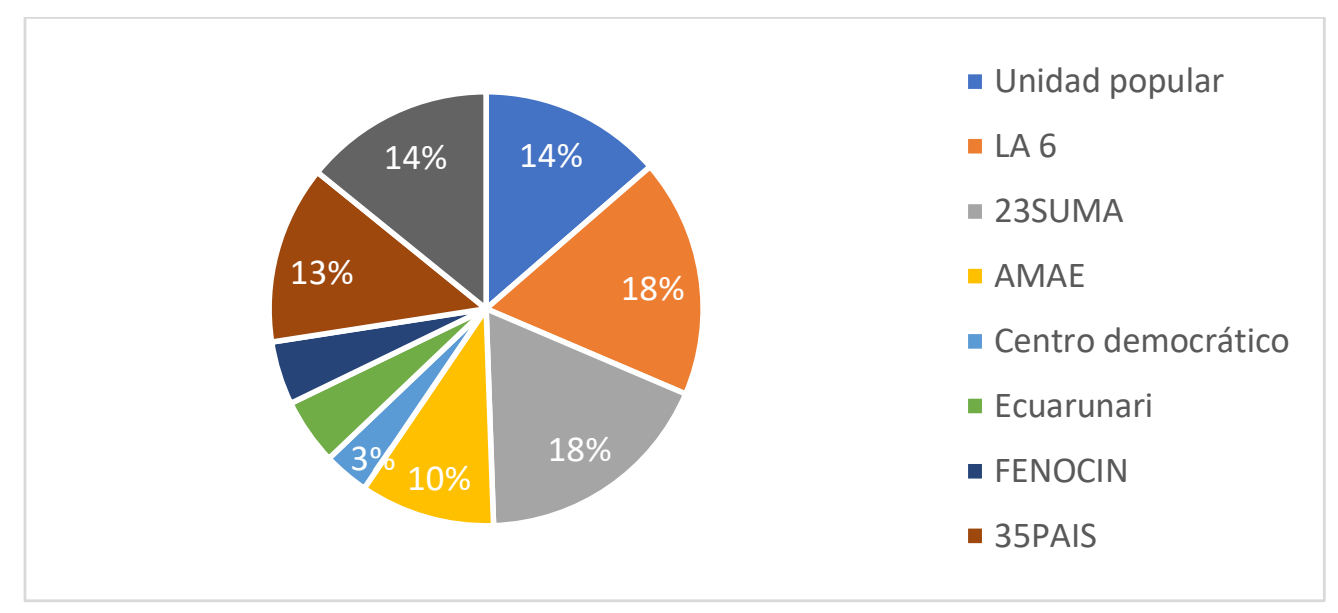

Fuente: Elaboración propia 
Por otro lado, grupos mas pequeños en redes, como la Confederación Nacional de Organizaciones Campesinas, Indigenas y Negras (@fenOcin), así como la Asociación Mayoritaria de Afrodescendientes del Ecuador (@AMAE), cuyas cuentas no cuentan con tal relevancia o seguidores, intentaron basar su estrategia en la mención de otras cuentas, destacandose cada una con 1288 y 494 menciones respectivamente. En concordancia, al revisar el número de hashtags que contenía cada una de las cuentas, destacaron @ecuarunari y @unidadpopular, pues es un buen mecanismo para llamar la atención de quienes están buscando tendencias y desean conocer más cuentas que hablan de un tema. La herramienta también permitió conocer cuántas cuentas habían tenido interacción en forma de respuestas y la única que destacó del listado fue centro democrático, con apenas 63 respuestas de los 800 tweets que realizaron a lo largo de la campaña electoral, seguidos por 44 respuestas de @AMAE, representando el 55\% de toda su gestión y 25 de @fenocin, con un $27 \%$ de representación, como se muestra en el gráfico.

Gráfico 4. Tweets en respuesta a otros tweets.

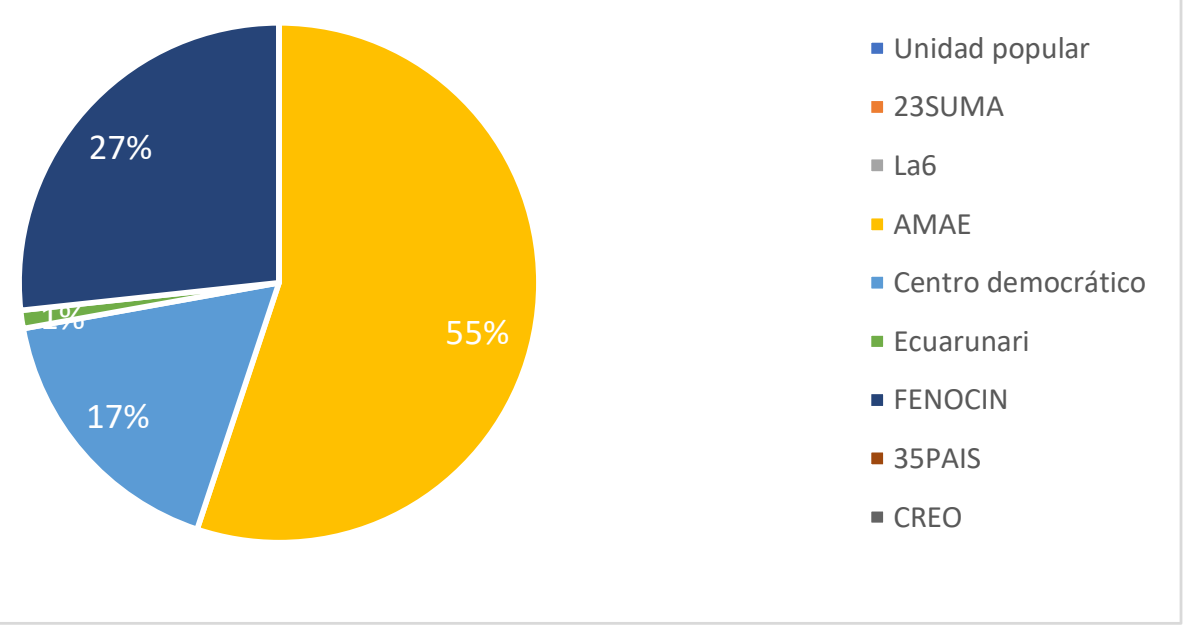

Fuente: Elaboración propia

A partir de estos resultados, se comprueba que solamente 4 de todos los partidos y movimientos con cuenta en Twitter han considerado realizar interacción con otros usuarios. 


\section{CONCLUSIONES}

Las conclusiones obtenidas en la presente investigación no difieren en gran medida a las que se plantearon con el modelo LOOP en la investigación de las elecciones presidenciales de Ecuador de 2017, pues aún se denota un estado neófito referente al uso de los medios digitales por parte de las asociaciones, esta vez no siendo acopladas a la representación de una figura política concreta sino reforzando una desición de consulta popular con dos posibles resultados, sí o no.

A partir de esta desición, y tomando en cuenta las facilidades de los formatos digitales que nos ofrecen las redes sociales como Twitter, han surgido a la vez nuevas, se debería suponer que varreras de escucha entre los ciudadanos y los partidos que resultaban comunes hace unos años se habrían abolido. Sin embargo, esta situación persiste, no por el hecho de que no se puedan usar los medios de respuesta, sino por la característica predominante de usar a las redes sociales como un canal más de difusión y no como un medio de escucha activa. El caso de las elecciones de Ecuador no fue diferente, y muchos usuarios buscaron generar intervención, pero esta no se dio, por falta de protagosnismo de las redes sociales, con un uso muy limitado, regularizado solo en 8 cuentas de partidos y movimientos, de 40 inscritos para la campaña electoral, y con un reflejo firme en la casi nula tasa, tanto de respuestas a mensajes, como de espacios de interacción en la mayoría de los casos.

Queda claro que cada espacio en línea brinda una oportunidad, en este caso a las propuestas de Sí o No para una consulta, a llegar a una masa de personas que utilizan diariamente de manera plural las redes sociales, pues, aunque aún no sean un determinante al momento de realizar una desición de voto, cuentan como espacios de información y debate con un público que decide en base a la atención que recibe. Sin embargo, al ser una campaña apoyada por 36 partidos con una postura y solo 4 de contraparte, no se lograron evidenciar de manera robusta errores al momento de usar Twitter pues, mientras algunos partidos no generaban contenido continuo o de interés, otros si mantenían la atención del público, cubriendo a los primeros.

Finalmente, se puede concluir que la participación política en las redes sociales no se contempla como eficaces al momento de enfrentar un periodo de campaña. No lo fueron para las elecciones presidenciales y tampoco para la consulta popular de este año, pues aún no se toma en cuenta el espacio virtual como determinante. 


\section{REFERENCIA BIBLIOGRÁFICA}

Trottier, D., \& Fuchs, C. (2015). Introduction. En D. Trottier, Social Media, Politics and the State: Protests, Revolutions, Riots, Crime and Policing in the Age of Facebook, Twitter and YouTube (págs. 4-16). New York: Routledge.

Mandiberg, M. (2012). Mecanismos. En N. Y. University, The social media reader (págs. 120). New York: New York University Press.

Marquina, J. (2012). Diferencias entre comunidad y red social. En J. Marquina Arenas, Plan social media y community manager (págs. 14-16). Barcelona: UOC.

Caldevilla, D. (2010). Las Redes Sociales. Tipología, uso y consumo de las redes 2.0 en la sociedad digital actual. Documentación de las Ciencias de la Información, 33, 65.

Interactive Advertising Bureau. (2017). CONSUMO DIGITAL ECUADOR 2017. Recuperado el 08 de 2018, de IAB Ecuador: http://iabecuador.com/doc/EstudioDigital2017_IAB.pdf Coronel, G., \& Mier, A. (2011). IMPACTO DE TWITTER EN ECUADOR, CASO $30 S$. Recuperado el 09 de 2018, de Academia.edu: https://www.academia.edu/8188504/IMPACTO_DE_TWITTER_EN_ECUADOR_CA SO_30S_CISCI_2011

Escandón, P. (2013). Política Electoral Y Política Ciudadana: Partidos, Movimientos Sociales Y Anticandidatos En La Campaña Preelectoral Del Ecuador. Razón y Palabra, 17(1_18), 246 - 255.

Pulla, G. (2017). CIBERPOLÍTICA EN ECUADOR 2017: ANÁLISIS DE LA CAMPAÑA PRESIDENCIAL EN REDES SOCIALES, WEBS Y BLOGS. Málaga, Andalucía, España.

Small, T. (Otoño de 2010). Canadian Politics in 140 Characters: Party Politics in the Twitterverse. Canadian parlimentary Review, 33(3), 39.

Marín, I., Punin, M. I., Hinojosa, M., \& Ruiz, J. (Abril - Junio de 2016). Twitter como herramienta estratégica en la política ecuatoriana. Razón y Palabra, 20(93), 120 - 134.

Albornóz, M. B., \& Rosales, R. (30 de 10 de 2012). Periodismo ciudadano y Twitter. El caso del 30-S ecuatoriano. Versión Estudios de Comunicación y Política, 30. Recuperado el 09 de 2018, de Flacso Andes: http://www.flacsoandes.edu.ec/sites/default/files/\%25f/agora/files/periodismo_ciudad ano_y_twitter.pdf

Almansa, A. (2015). Ciberpolítica en España. Parlamentario en la red en las dos últimas legislaturas. 8. 
El Comercio. (19 de 01 de 2018). ¿Cuáles son las siete preguntas del referéndum y la consulta popular del 4 de febrero de 2018 en Ecuador? El Comercio, págs. https://www.elcomercio.com/actualidad/preguntas-consulta-referendum-leninmorenoecuador.html. 